Cultures \& Conflits

18 | été 1995

La violence politique des enfants

\title{
Les enfants et la violence politique
}

\author{
Louis-Jean DUCLOS
}

\section{(2) OpenEdition}

Journals

\section{Édition électronique}

URL : http://journals.openedition.org/conflits/469

DOI : 10.4000/conflits.469

ISSN : $1777-5345$

Éditeur :

CCLS - Centre d'études sur les conflits lilberté et sécurité, L'Harmattan

Édition imprimée

Date de publication : 15 juillet 1995

ISSN : 1157-996X

Référence électronique

Louis-Jean DUCLOS, «Les enfants et la violence politique », Cultures \& Conflits [En ligne], 18 | été 1995, mis en ligne le 04 mars 2005, consulté le 30 mars 2021. URL : http://journals.openedition.org/conflits/ 469 ; DOI : https://doi.org/10.4000/conflits.469

Ce document a été généré automatiquement le 30 mars 2021.

Creative Commons License 


\title{
Les enfants et la violence politique
}

\author{
Louis-Jean DUCLOS
}

1 «Comme ils sont plus jeunes, il n'en seront que plus impitoyables» (Apologie de Socrate)

2 Les débats préparatoires à la conception de ce numéro ont porté d'emblée sur la pertinence du thème proposé : les enfants sont-ils susceptibles d'un comportement politique sans cesser d'être enfants? Si oui, leur violence politique présente-t-elle un caractère spécifique? La discrétion de la littérature scientifique, sinon romanesque, à cet égard faisait en effet douter que la violence politique des enfants fut un vrai sujet. La première de ces questions en cache en réalité une autre, occultée par une incertitude langagière allègrement encouragée par la pratique sociale et politique : qui est enfant? A l'évidence l'enfant à la mamelle a peu à voir avec celui qui, adolescent, hante un champ de bataille ou casse des vitrines. Si l'on sait sans trop d'ambiguïté comment et quand on entre en enfance, encore que le débat sur l'avortement en fasse douter à la marge, notre connaissance des modalités de sortie reste floue, notamment sur ce qui se passe, socialement parlant, entre le commencement et la fin du parcours pré-adulte? Annick Percheron dans son ouvrage fondamental sur la socialisation politique dans l'enfance et dans l'adolescence a bien indiqué que, comme la maturation biologique, l'émergence sociale, donc politique, des enfants était progressive. Ce serait alors par commodité que les sociétés se sont plues à distinguer dans ce continuum des seuils ponctués de rites plus ou moins initiatiques, jalonnant, après l'accès à la vie, celui de l'«âge de raison », de la puberté, de l'entrée, enfin, souvent par degrés, dans la cité des adultes elle-même. S'il n'y a pas de limite d'âge inférieure qui place l'enfance hors du champ politique en tant qu'objet - massacre et rafles d'enfants en bas âge l'attestent - il n'en va pas de même pour qu'elle s'y comporte en sujet, ce qui seul ici intéresse. Certes la socialisation des non adultes en général et leur éducation en particulier, visant à la reproduction sociale et politique, traitent les enfants en sujets virtuels et acteurs différés d'une vie politique dont ils auront à observer les règles. Mais dans l'immédiat on leur enseigne surtout, et on leur impose, leur exclusion en tant qu'enfant de toute initiative politique, exclusion destinée dans maints systèmes à s'appliquer au plus grand nombre et toute la vie. Cette règle de l'exclusion politique juvénile fonctionne si bien dans nos sociétés que, jusqu'à présent, la présence de l'enfant sur la scène sociale 
en tant qu'acteur politique demeure une " anomalie » que les sciences humaines ont négligé d'explorer. La définition de son autonomie d'acteur social est encore à construire. Sans doute s'est-il trouvé des socio-politologues pour s'en expliquer, arguant en particulier du fait que la qualification d'enfant ne définit pas une catégorie sociale déterminée. Constatant que l'accession à la maturité s'opère sans rupture à partir d'une dépendance maternelle absolue jusqu'au degré parfait de l'autonomie alimentaire, affective et intellectuelle, on devrait en déduire qu'il est artificiel et peu scientifique de découper ce continuum en périodes. C'est évidemment à la fois vrai et erroné : vrai qu'il n'est pas de seuil historique précis de passage d'un stade de maturation à un autre; erroné de refuser la réalité de ces différents stades à l'égard desquels, selon Annick Percheron, l'âge représente une variable-clef ${ }^{1}$. Biologie oblige, serait-on tenté d'écrire, productrice de critères intrinsèques individuels. L'autonomie gestuelle et la capacité de discernement, liées toutes deux au développement physique de la personne, en font partie. Dès lors que l'enfant a la capacité d'actions raisonnées, rien n'empêche a priori, que celles-ci soient violentes et orientées à des fins politiques, parfois très antérieurement au temps de l'adolescence. C'est affaire de surdéterminations sociales fortement marquées, évidemment, par les histoires collectives mais aussi individuelles.

3 L'enfance politique : les limites de l'exclusion Biologie, culture et circonstances se combinent donc pour tracer, sinon les limites exactes, du moins les étapes d'une maturation politique, dans laquelle l'expérience prouve justement que la violence politique joue un rôle important, comme si la manière d'être politique des enfants ne pouvait être que violente, et comme si, à l'inverse, la violence était l'une des productions sociales majeures de l'enfance et que sa politisation, à défaut d'être très "morale ", lui donnait au moins du sens. En temps de crise et plus communément sous d'autres cieux, il n'est pas d'âge si tendre - à condition qu'il soit de raison - qui ne soit sollicité de participer à la violence politique ambiante. A l'autre extrémité chronologique de la socialisation politique juvénile et dans nos sociétés, on retrouve aussi le recours au rite de sortie du service militaire, symbole lui aussi de violence politique. Entre ces deux termes, l'entrée en participation précoce à la violence politique et ce qu'un large consensus d'opinion considère comme la sortie de la minorité civique, la représentation adulte des jeunes oscille entre celle du faible à protéger et celle de l'irresponsable à surveiller. Les hésitations du droit qu'on a toujours intérêt à consulter en pareille matière rendent compte de cette approche contradictoire. Voici longtemps en effet que le droit positif tant interne qu'international s'efforce de fixer les étapes du passage à l'âge adulte, réputé celui de la pleine capacité. Le droit humanitaire, en particulier, a découvert tardivement qu'une participation des enfants aux conflits armés internes et internationaux justifiait qu'ils bénéficient dans cette situation d'une protection spéciale. Dernière en date, la convention des Nations unies relative aux droits de l'enfance fixe non sans hésitation cette sortie d'enfance à quinze ans ${ }^{2}$. Les droits internes nationaux, en distinguant plusieurs sortes de majorités, ont nettement indiqué eux aussi que l'enfance était un temps d'irresponsabilité et d'incapacité légales à géométrie variable dont on ne sortait en tout cas que par degrés. Ils ont même prévu que vieillards et autres malades pouvaient y replonger et retrouver leur statut $d^{\prime}$ 'incapacité initiale ${ }^{3}$, sanctionnant de ce fait leur régime d'exclusion sociale. C'est également à maints égards un régime d'exclusion que celui de l'enfance, mais la nature est loin d'en être la cause unique. A vrai dire, celle-ci dit son dernier mot avec l'entrée des individus dans l'âge 
physiologiquement nubile. Tout le reste n'est que contrainte politique habillée de conventions. Si l'on pense avec certains politologues désabusés que le but du pouvoir est de se conserver, de s'étendre, mais aussi de se concentrer, on comprend bien l'intérêt des gouvernants à retarder au maximum l'entrée des jeunes, comme celle des femmes naguère, des pauvres, des étrangers et autres marginaux, dans le débat politique réel et dans les cercles de la décision. C'est pourquoi aussi, au nom de l'idéal démocratique, des sociologues qui ne s'embarrassent pas de conservatisme, comme Alain Touraine, préconisent d'abaisser le vote des jeunes de dix-huit à quinze ans en raison du fait, dit-il, qu'à défaut d'être "constitués en acteurs politiques " ils représentent "une part importante du marché » et «du chômage " ${ }^{4}$. A ce débat près sur la "limite d'âge» de la subordination sociale absolue et de l'irresponsabilité politique, les détenteurs du pouvoir et les conservateurs du système politique et social global sont d'accord pour voir dans les « jeunes » une « classe dangereuse » justiciable de l'«exclusion " ${ }^{5}$. Pire, ils ne sont pas seulement dangereux en tant que "classe » mais, selon Charles Melman, en tant qu'individus, faute d'« intérioriser les limites posées par notre culture aux manifestations de la violence». Leur "efficacité criminelle » est d'autant plus grande qu'ils bénéficient en général de l'«impunité attachée à leur âge " ${ }^{6}$. C'est pourquoi la société des adultes, toute à la fois soucieuse de sa sécurité propre et de sa propre reproduction, voit dans la politisation des jeunes un «désordre " attentatoire à la tranquillité publique, mais aussi à l'ordre politique en vertu duquel le monopole de la violence légitime revient à l'autorité politique qui, dans un société équilibrée, ne saurait être que celle des forts, donc des grands. Pour moraliser le tout, notre sensibilité moderne a inventé le concept de l'innocence enfantine que certaines sociétés, ailleurs, et les experts, chez nous, rejettent. Psychologiquement irresponsables, ils ne le sont pas pour Marcel Czermak qui les voit au contraire capables de finalisation et de stratégie ${ }^{7}$. Si, ajoute-t-il, leur violence, politique ou non, est refoulée sous la pression du social et de l'inconscient, qui sont selon lui les deux faces, interne et externe, d'un même phénomène, c'est celle-là même qui resurgit chez l'adulte, habillée d'une idéologie qui n'a d'autre fonction que la méconnaissance de nos pulsions. Violents "par nature " au point qu'il leur arrive de tourner contre eux-mêmes leur violence, les enfants doivent être empêchés de nuire ; innocents, ils méritent d'être protégés ${ }^{8}$. Un encadrement social ad hoc produit ce double effet répressif/éducatif redondant. La sollicitude parentale, en réalité fort variable, en est le premier stade qui enferme ses enfants le plus légitimement du monde dans un encadrement social de proximité fort, complexe et parfaitement inhibiteur. Ainsi, plus encore que la loi, un sentiment de crainte, de révérence et d'affection réussit, d'une manière générale, à retenir dans le cercle de famille, sur les bancs de l'école, à l'atelier d'apprentissage, dans les stades et mouvements de jeunesse $\mathrm{du}$ dimanche, politiques ou non, une enfance aux nuisances neutralisées. Paradoxalement en apparence, cette "éducation" est aussi une technique d'infantilisation qui permet à la classe politique installée de prolonger le temps de l'exclusion des jeunes. Elle recule le temps des contestations, elle permet aussi de commodes mobilisations instrumentales. Quel que soit le projet pédagogique proclamé, une infantilisation plus ou moins sophistiquée déborde largement le cadre restreint de la jeunesse et on la voit partout à l'oeuvre, dans les sociétés religieuses et militaires, chez les militants des syndicats et des partis politiques, dans la main d'oeuvre des entreprises, le milieu scolaire et étudiant, les consommateurs de toutes espèces, tout particulièrement de la production télévisée. Dociles et enthousiastes jusqu'à un certain 
point, disposant d'un potentiel de violence peu entamé, ces enfants représentent aux mains des entrepreneurs politiques légitimés une ressource que ceux-ci ne répugnent pas à mobiliser à condition d'en garder le contrôle.

4 La manipulation politique de la violence juvénile Classe «dangereuse » dès qu'elle est musclée, les enfants ne vont pas néanmoins sans offrir aux entrepreneurs politiques quelque concours. Fragiles, sans défense ni défenseurs, ils font merveille parait-il, quand l'ambiance s'y prête, dans la fabrication des tapis pakistanais dès l'âge de quatre ans ${ }^{9}$. En politique, un mélange de non-discernement relatif, d'ardente disponibilité et de déférence bien organisée, fait de l'adolescent, l'instrument idéal d'une violence publique supplétive. Sans battre en brèche le principe d'une exclusion juvénile qui les fragiliserait, les entrepreneurs politiques recourent depuis l'antiquité à l'enrôlement des jeunes. Ceux-ci sont censés alors remplir, entre autres, une fonction militaire. A court d'effectifs les états-majors les enrôlent pour compenser l'hécatombe des vétérans ou corriger le déséquilibre initial des forces. Il y aurait aujourd'hui deux cents mille « enfants-soldats » de par le monde. Parfois embrigadés comme d'ordinaires troupiers dans les unités régulières, ils sont plus à leur place dans les groupements de supplétifs. De la sorte, ils risquent moins de susciter par leur inexpérience et leur indiscipline l'agacement des professionnels. D'autre part, réguliers ou supplétifs, ces grands enfants-là, sont plutôt moins exigeants que leurs aînés. Plus prompts aux exaltations sacrificielles ou sensibles, quoi qu'ils disent, à la hiérarchie des âges, ils posent notamment moins de questions sur le bien fondé de leur immolation programmée. Leur présence au front enfin peut être exploitée par les gouvernements de guerre pour l'exaltation émotionnelle de l'arrière et, sans grande chance de succès, pour faire peser sur l'ennemi le chantage du bouclier humain. Quoi qu'il en soit, ces mobilisations d'enfants dénotent une situation stratégique compromise. On ne sache pas que l'envoi au front des " Marie-Louise », des "Pupilles de la Commune », des dernières " Jeunesse hitlérienne », ou des «bassiji » iraniens aient changé l'issue des combats. A l'inverse des violences " économiques » faites à l'enfance, les «violences d'Etat " dont celle-ci serait spécifiquement la cible, quoique rarement combattues, sont aujourd'hui sévèrement jugées par la «communauté internationale» dans la mesure où elle partage ou affecte de partager une "éthique occidentale», cependant que se poursuivent et se développent, dans des situations qu'on se plaît à croire exotiques, des enrôlements forcés de jeunes enfants dans des centres d'esclavage civil ou militaire à la manière mamelouk. Paradoxalement, si ces pratiques sont susceptibles d'indigner les parents au demeurant impuissants, ce ne sont pas celles qui induisent le plus fréquemment une révolte active des dissuadés, résignés, voire, à l'inverse, littéralement déchaînés, fanatiques sanguinaire ${ }^{10}$. Mobilisables aussi, les enfants le sont, en période révolutionnaire, par des acteurs qui, pour être parfois clandestins, n'en sont pas moins de véritables institutions, même si, dans des situations apparemment anarchiques, la dimension des unités d'organisation politique se rétrécit. On observera que les enfants enrôlés plus ou moins de force, apprécieront de ne pas paraître tels. Tout d'abord instruments passifs d'une entreprise dont la maîtrise leur échappe totalement, l'esprit vient rapidement aux adolescents d'une interactivité politique dans le cadre de laquelle ils ne sont dépourvus ni d'initiative ni d'efficacité, manifestant pour le moins un désir de changement de leur cru. Quel «ado " ne souhaite en effet, notamment lorsque son bilan amour/contrainte penche en faveur de la seconde, changer les règles du jeu social, abolir le carcan familial, l'esclavage du travail, la répression de l'école, le commandement des sergents? Les commissaires de la révolution ont donc beau jeu de 
susciter des subversions qui ont des airs de vacances, de vengeance et de revanche. Ils ne s'y emploient cependant qu'avec circonspection. Avant le renversement du régime, le jeu est dangereux et les perspectives médiocres; ils ne s'y risquent guère. C'est une fois en place que le gouvernement révolutionnaire ou le petit chef de guerre local mobilisera ses jeunes pour parfaire ici quelque destruction de l'ordre social ancien ou mener ailleurs une manoeuvre purement politicienne. Cette instrumentalisation du potentiel révolutionnaire de l'adolescence, libéré par les situations révolutionnaires elles-mêmes, est parfaitement illustrée dans les articles que Jacques Andrieu et Farhad Khoroskhavar consacrent respectivement ci-après à la Chine et à l'Iran. Dans les deux cas on voit les gérontes qui gouvernent, le Timonier et le Guide, qualifiés par Khoroskhavar de "superadultes", recourir aux services des sous-adultes pour combattre les adultes. Ainsi voit-on les Gardes Rouges chinois télécommandés de Pékin s'en prendre à l'appareil du régime, première mouture, et les Bassiji iraniens, répondant aux consignes de Qom, s'en prendre aux vestiges de l'ordre social ancien. Producteurs de violence politique contre l'étranger ou contre l'ennemi de l'intérieur, dans les rangs d'une armée régulière ou d'unités supplétives, de milices en uniformes ou de groupes militants plus ou moins clandestins, ces enfants sont littéralement en service commandé. Il n'est pas sûr que la violence politique qu'on leur a donné licence de produire soient très différente dans ces modalités de celle que les situations de guerre libèrent chez leurs aînés. Agissant sous contrôle, ils ne demeurent pas moins des exclus de la politique, tant, du moins, que ce contrôle subsiste. La violence politique juvénile autonome L'infantilisation a ses limites. Les jeunes enrôlés, s'ils survivent, ont vocation à devenir anciens combattants et vétérans, ce qui nous renvoie à un autre sujet. Mais il est d'autres cas de figure, à vrai dire exceptionnels, qui font l'originalité de ce débat :la violence politique autonome des enfants. Constatons d'abord qu'elle est possible. Lorsque Bernard Thomas nous raconte dans la Croisade des Enfants ${ }^{11}$ (1212) comment son héros, Etienne de Cloyes (13 ans), un soir de carnaval, entraîne son petit monde sur la route de Constantinople au grand dam des mères, des curés et des seigneurs, c'est bien de cela qu'il s'agit. Le tambour Bara et l'enfant-grec-aux-yeuxbleus étaient assurément des volontaires, échappés de structures sociales bouleversées par des crises patriotiques. La pugnacité des jeunes catholiques irlandais du Nord, n'est pas réductible à l'engagement de leurs parents; elle relève d'une "youth culture " spécifique $^{12}$. On verra plus loin avec Frédéric Chauvaud que Gavroche appartient à une société juvénile au service du projet républicain des adultes sans pour autant être manipulé par eux et que ses successeurs ont à leur actif, au XIXème, plus d'une « insurrection buissonnière » incontrôlée. Quant aux jeunes palestiniens de l'Intifada, Sylvie Mansour ne souligne pas moins leur autonomie en évoquant la difficulté des adultes à s'adapter à ces enfants et la situation de ces adolescents qui tout à coup réagissant et manifestant, revendiquant par là un changement de statut. Une subversion aussi inouïe de l'« ordre des choses » n'est pas possible sans la réunion de quelques conditions. La première, comme dans toute révolution, est une prise de conscience de graves frustrations ou de sévères brimades que les inhibitions résultant d'un maternage prolongé, des conditionnements de l'idéologie dominante, de l'abrutissement du travail forcé, avaient jusqu'alors fait passer pour inévitables et rendu de ce fait supportables, jusqu'aux limites du martyre. Que tombent en ruines l'institution familiale et autre encadrement spécifique de la population enfantine, celleci, qu'aucune révérence ni crainte ne retiendront plus au foyer, à l'école ou à l'atelier auront vite fait de se répandre dans les champs et dans la rue pour se lancer comme au 
jeu dans des batailles inventées ou appropriées. L'expérience et l'imagination aidant, ils anticiperont la ruine des morales anciennes ${ }^{13}$, fantasmatiseront de démoniaques adversaires, se fabriqueront des idoles patrimorphes et des fratries d'élection, ardentes et tragiques, s'enivreront de rêves et de pratiques d'aventures et de sacrifices extraordinaires jusqu'à épuisement de leurs énergies, par la mort, l'échec, ou la fatigue, à moins qu'elles ne soient récupérées par les adultes que, de toute façon, les survivants deviendront eux-mêmes. Sur tout ce parcours, la marge risque d'être étroite entre violence politique et délinquance crapuleuse. Elle ne l'est pas plus que dans la qualification de la violence révolutionnaire où l'intentionnalité demeure une référence cachée et où l'appartenance à une organisation légitimante n'apparait, éventuellement, qu'après coup, selon les fortunes de la compétition. Ce cas s'applique particulièrement bien aux « casseurs » de nos manifestations; ne sont-ils que des délinquants de fait, une "chienlit», disait de Gaulle, ou déjà des politiques comme le disent à leur place certains dirigeants de " coordinations » par ailleurs " comme-il-faut »? Les «bandes ", gangs et groupuscules qui sévissent à Harlem ou contribuèrent à désoler Beyrouth relèvent d'un même mouvement, qu'on dit pré-politique par référence à son organisation rudimentaire, alors qu'il est bel et bien politique dans ses motivations et objectifs. N'empêche, il faut une bonne dose de désencadrement social, de dislocation politique et, plus trivialement, d'incapacité policière pour que s'effondrent les systèmes parentaux ou quasi-parentaux qui produisaient jusqu'alors le contrôle des conduites des jeunes, neutralisaient leur dangerosité, leur prodiguaient un châtiment éducatif socialement accepté et même psychologiquement désiré par les immatures qui le subissaient; un système qui, pour être cohérent, postulait l'incapacité politique des jeunes et l'organisait. Une violence politique précoce, caractérisée par un minimum d'auto-organisation, ne se conçoit donc que dans le cadre de situations révolutionnaires, pré-révolutionnaires ou quasi-révolutionnaires, ménageant des « niches » d'anarchie d'où émerge le « héros » de ce numéro : le non-adulte produisant une violence concertée à finalité politique. Tout au contraire du cas de violences juvéniles téléguidées qui confirment le statut subordonné de l'enfance, la violence politique autonome des jeunes exprime une rupture, un défi à l'ordre public et au contrôle social, toutes institutions confondues: Etat, puissance occupante, autorités traditionnelles d'une part, famille, appareils scolaires, employeurs d'autre part, se délégitimant plus ou moins simultanément, par excès de brimade, ou par carence. Dans tous les cas on observera que, si la violence des enfants peut n'être pas politique, leur irruption en politique en tant qu'acteur autonome ne peut pas ne pas être transgressive et violente. Elle est inconcevable, comme semble le prouver a contrario l'article de Guy Nicolas sur l'agitation politique des jeunes nigérians, dans une société aux structures traditionnelles, plus ou moins patriarcales, fortes ${ }^{14}$. Contrevenant au statut de l'enfance, elle s'inscrit nécessairement dans un ensemble social déstructuré faute de quoi les enfants n'auraient ni l'idée ni les moyens de contester leur statut d'irresponsabilité et de soumission qui, dans les sociétés les plus avancées, passent pour être lié au temps de l'éducation et ressortir aux règles du contrat social. Que survienne l'étranger envahisseur, bousculant l'organisation civile, politique et policière, recourant inévitablement à des opérations de répression ou de représailles globales, un mouvement de résistance "nationale" se généralise. S'il advient alors qu'après l'élimination des contestataires les plus audacieux, la classe des adultes découragée se résigne et se soumette ou, au mieux, se réfugie dans une résistance mentale d'attente, l'imprudence, ou l'inconscience, ou le romantisme des enfants conduisent ceux-ci, 
comme le montre le cas palestinien à s'avancer dans le désert politique créé par la prudence des "grands" et des "vieux ». Ici la misère politique des aînés favorise l'entrée des «z'enfants de la Patrie (...) dans la carrière ». Survivant aux ruines ou au chômage de l'école, de l'emploi et, finalement de la famille à l'encadrement desquels l'enfant est normalement assigné, celui-ci, "libéré ", tend à autodéterminer sa conduite, à recomposer son environnement social et à s'inventer un projet et une organisation politique. Le fait, en particulier, de plonger des classes d'âge entières dans l'oisiveté, de les jeter littéralement sur le pavé, et de les rendre disponibles pour toutes sortes de mobilisations compose les conditions idéales d'une entrée de groupe dans la violence politique. Cependant le désencadrement social des enfants n'est pas forcément lié aux effets d'une oppression extérieure. Il est d'autres voies plus insidieuses pour la déligitimation des institutions qui maillaient leur espace. Que l'école se disqualifie par l'inadéquation de ses programmes, de ses méthodes, par son inutilité sociale, voire par sa nuisance, que, simultanément le travail des enfants apparaisse comme odieux ou seulement superflu, qu'enfin la fonction parentale se dévalorise par auto-démission ou par déconsidération sociale de ses titulaires, ou par l'effondrement de ses références idéologiques, nous retrouvons le non-adulte dans une situation d'émancipation de fait, l'entraînant à contester le statut d'incapacité politique dans laquelle il était enfermé. Du coup l'idéologie traditionnelle dominante qui structurait le discours politique et peu ou prou la conduite des adultes se trouve remise en question. L'éventualité alors apparaît, comme en «mai soixante-huit » d'une mise entre parenthèses plus ou moins prolongée des pouvoirs publics et civils légitimes. Sur la scène vidée de ses acteurs « légitimes » les jeunes générations tenteront de faire mieux que leurs prédécesseurs si elles en conservent le modèle idéologique, soit de faire autrement s'ils érigent la révolution culturelle en système. Dans tous les cas elles caresseront l'utopie d'une nouvelle planète. L'entrée prématurée dans la cité interdite du politique, avec tout ce qu'elle comporte de subversion des hiérarchies, de pratiques légitimées de la violence, de projections imaginaires, ouvre devant les enfants émerveillés un champ inespéré de vacances en vraie grandeur, de sport, de jeu, de libre cruauté et d'innovation. Il n'y a pas lieu d'attendre qu'une violence politique juvénile pure, c'est-à-dire celle dans laquelle les consignes des adultes n'interfèrent pas, s'exprime de manière très différente de celle de leurs aînés si ce n'est par un déficit d'institutionnalisation; ou encore qu'une violence politique d'adulte désinstitutionnalisée, libérant les «instincts", soit très différente de celle des enfants s'il est vrai, comme l'affirme Marcel Czermak, qu'elle n'est que la résurgence de violences juvéniles refoulées. En retenant par exemple quelques couples de pôles de conduite entre lesquels oscillent la conduite des violents politiques tels que ludique $\mathrm{c} /$ sadique, mimétique $\mathrm{c} /$ inventive, prédatrice c/ idéologique, déclamatoire c/ meurtrière, c'est à peine si on peut légèrement privilégier le ludique, notamment dans les phases initiales de la geste violente. Bernard Thomas, à propos de la Croisade des enfants émet l'hypothèse que celle-ci aurait commencé comme un carnaval qui eût dégénéré. Angelina Peralva montre ci après que nos "loubards" de banlieue prennent un certain plaisir à exprimer violemment une critique quasi-politique de la situation dans laquelle ils se trouvent. Cependant, généralement, advient le temps de la triste épreuve des luttes prolongées. Epuisement physique et accès de découragement, mort, blessures et captivité, revers certains contre succès précaires, auront tôt fait de dissiper les ivresses de groupes et le souvenir même des aubaines des débuts. Le monde des adultes, lui, où se recrutent l'adversaire et les alliés de l'adversaire, est toujours là. Les ressources 
biologiques et culturelles d'un monopole politique protéiforme adulte demeurent intactes. Il n'aura finalement été que marginalement et passagèrement contesté par l'irruption des jeunes.

5 La fin des violences politiques juvéniles d'enfants La violence politique des enfants n'a pas d'avenir. Aucun de ces enfants-combattants, engagés les armes à la main ou la menace à la bouche dans un projet politique de leur cru ou enrôlés par des politiciens professionnels, ne survivra en tant que tel. Les uns, recrus de fatigues et quelque part estropiés, rentreront discrètement à la maison ou se découvriront des pénates d'adoption. Certains se perdront à jamais dans un paysage embrouillé. D'autres se trouveront récupérés par des pouvoirs qu'ils auront éventuellement combattus. Bon nombre d'entre eux, à titre de rachat ou de récompense se verront offrir du service dans de très institutionnelles unités militaires ou para-militaires en tant que "préadultes (...) sous la tutelle des adultes » (F. Khoroskhavar). Les plus malchanceux seront morts en cours de route. Même victorieux, les survivants juvéniles d'une guerre étrangère ou d'un conflit civil ne fonderont une "république des enfants", dotée des attributs de la puissance publique, autonome et armée, qu'à titre de parenthèse précaire et fugace, insupportable aux adultes, qui n'auraient de cesse qu'ils l'aient liquidée ou réduite à un simulacre momentané. Pire encore, à supposer qu'elle corresponde au projet politique des jeunes militants, elle ne serait pas moins menacée de l'intérieur par l'inexorable vieillissement des chefs. Adultes à leur tour, admis ou s'imposant dans la compétition politique globale de la cité, la militance juvénile n'aura en somme été pour eux qu'un raccourci, leur ENA en somme, pour aborder aux plages où le pouvoir se partage ou se confisque. Soyons assurés qu'une fois installés, ils ne seront pas les moins habiles à apprivoiser la violence politique des enfants pour l'instrumentaliser peut-être, mais plus probablement pour mieux la liquider ou, tout au moins, en éliminer au plus tôt toute trace d'autonomie véritable. Il n'y a pas d'exemple historique où la violence politique juvénile ait été une référence sérieuse et durable, tant il est vrai qu'elle ne trouve pas place dans l'organisation politique des systèmes post-barbares d'après-crise. La récupération apparaît alors comme une étape de son éradication. Avant d'en arriver là, la violence politique des enfants, expression d'un désordre social et politique, aura cependant montré qu'elle n'est pas dépourvue d'efficacité immédiate sur la scène politique globale. Etant d'abord un révélateur de crise elle pourrait être, pour les pouvoirs en place, un signal d'urgence, compte tenu notamment de son caractère profondément subversif et, selon la perception commune, pathétique et exemplaire. En fait ils la gèrent, non sans résultats, selon les méthodes éprouvées du réformisme démobilisateur. Toutefois, les rébellions politiques enfantines une fois installées, quoique négligeables en termes de puissance armée, ont sur la psychologie collective un impact considérable. Les risques courus par les enfants, que chacun a tendance à identifier approximativement aux siens, suscitent l'angoisse, mais aussi avec l'absurde fierté des mères et la confusion des aînés, entraînent, stimulent ou relancent la mobilisation des collectivités. L'opinion, qu'émeut le sacrifice de Gavroche, a tôt fait de l'ériger en modèle et on assiste alors à ce curieux retournement où, le mimétisme changeant de camp, la fonction pédagogique passe des adultes aux enfants. Leur violence politique, ne serait-ce que parce qu'ils en sont les premières victimes, joue ainsi un rôle mobilisateur parfois décisif dans la survie des idéologies de résistance, comme on le voit en Irlande du Nord et dans le territoire occupé de Gaza. Dans ce dernier cas, comme le montre Sylvie Mansour, c'est bien l'appropriation des mérites de l'Intifada par la direction politique de l'OLP qui a permis à celle-ci de se 
remettre en selle. Mais les enfants politiques, en tant que tels en tireront peu d'avantages. Ainsi que subsiste-t-il de l'essai, s'il n'est transformé par les politiques professionnels dans les rangs ou à la tête desquels auront pris place les surdoués du mouvement juvénile ? On peut vivre avec et c'est même le lot commun. II y a toujours à la marge de la société organisée où règne le monopole de la politique et de la violence légitime des adultes un résidu de violence politique juvénile indéracinée dont il suffit pour la tranquillité des dirigeants qu'elle soit cantonnée au plus bas des profils. Les frictions intermittentes entre ce qu'on pourrait appeler le «bloc de légalité » et la "sphère de marginalité " de nos sociétés d'abondance n'est en somme qu'un inconvénient mineur dont la routinisation même garantit aux marginaux la survie. Dans le cas où l'explosion de violence politique juvénile aura revêtu une certaine gravité elle laissera à défaut d'héritage du moins quelques traces. Que ces jeunes aient trouvé dans la pratique de la violence politique un élément de leur socialisation politique parait évident mais pour quel résultat au terme de combien d'avatars? De nombreux jeunes nazis sont devenus un demi-siècle plus tard de rassurants chrétiendémocrates. Bien des «anciens» de 1968, sur la lancée de leurs idéaux libertaires et égalitaires, ont atterri dans les rangs du néo-capitalisme le plus conforme. On peut supposer que chez d'autres la nostalgie le dispute à une lassitude désabusée, qui ne serait peut-être qu'un signe de rancissement. Mais l'histoire suit son cours avec ses anciens combattants et ses nouveaux enfants. Toute conclusion ne saurait être ici que provisoire. Du moins souhaitons-nous avoir posé un vrai problème de sociologie politique et indiqué qu'il y a bien une violence spécifique de l'enfance liée à des causes physiologiques (immaturité personnelle) ou sociales (exclusion). Ainsi nous a-t-il semblé que l'enfant encore "sauvage" pouvait être collectivement un acteur du politique sous certaines conditions biologiques, psychologiques, sociales mais selon des modalités qui, à la technicité près, n'étaient guère différentes des pratiques adultes. Peu performante, il nous a semblé encore que la violence politique des enfants n'avait qu'un impact limité sur l'évolution sociale et politique globale de la cité et sur le devenir des rapports internationaux. Le plus important reste sans doute que la violence politique des enfants, conçue et vécue comme production de contrainte concertée sinon collective, ordonnée à l'obtention d'un résultat politique, est envisagée et ressentie par tous les acteurs en cause comme une transgression de l'« ordre des choses ». Elle est à cet égard une "violence d'exception ». Troquant les contraintes du gynécée contre les risques du champ de bataille, et les candeurs d'une prétendue innocence contre le plaisir de faire souffrir, les intéressés y trouvent plus ou moins durablement le charme ludique de vacances indéfinies, le frisson de risques insolites et le (presque) tout permis d'un carnaval extrême. Stimulée par une sensibilité romantique, l'opinion y trouve peut-être un élément de sa nécessaire ration de symbolique et d'émotion mais la représentation commune de la société fondée sur le monopole politique des adultes, mâles de préférence, s'en trouve à peine perturbée. Certes la notion même d'enfant, créancier et débiteur d'obligations, reste sujet à discussion. Sa définition ne saurait se contenter d'être objective et substantielle, elle doit être aussi subjective et relative. Sont enfants dans une société donnée ceux que les adultes, gérants de cette société, considèrent comme tels. S'il advient qu'ils entrent en politique, ils n'en demeurent pas moins aux yeux du pouvoir adulte seul légitime des instruments ou des usurpateurs, non des partenaires ${ }^{15}$. Peu importe alors que, généralement pubères et physiquement performants, ils entrent dans la catégorie biologique et sociologique, peu pertinente de notre point de vue, de l'adolescence. On 
retiendra plutôt que du point de vue de la norme dominante, leur autogestion politique est scandaleuse, d'autant que, en butte à l'interdit des adultes, elle ne saurait être que violente. Ce n'est pas tant le fait qu'encore d'âge réputé tendre ils pratiquent et s'exposent à subir la violence politique qui choque, ni même qu'ils en soient un instrument manipulé par les "grands ", mais plutôt qu'ils l'exercent pour leur propre compte alors qu'ils en sont encore du point de vue du droit et de la coutume au stade de l'incapacité politique. C'est donc sans intentionnalité que nous avons parlé indifféremment de violence politique d'enfants ou d'adolescents ou encore de violence politique juvénile ou précoce. Comme on verra dans les articles réunis ici la violence politique des enfants est plus souvent manipulée que spontanée ou autonome. Dans le premier cas elle révèle que les dirigeants politiques en place auraient trouvé dans une politisation précoce des gisements de violence élémentaire investis dans l'enfance un renfort ou une diversion. Si, à l'inverse, la violence politique juvénile est autonome c'est que la classe politique adulte a laissé échappé son monopole politique ce qui est bien le signe que la subversion de l'organisation sociale, politique et idéologique du groupe y est totale. Dans ces conditions tout y est possible, y compris l'émergence d'un pouvoir d'enfants profondément révolutionnaire mais éminemment précaire. Rescapés éventuels d'hécatombes toujours possibles, les enfants sont néanmoins tous condamnés à terme par la transition des âges. C'est pourquoi les adultes s'attendent avec réalisme à voir ces jeunes gens venir les rejoindre pour la grande compétition universelle. Ils comptent bien, chacun pour son parti, bénéficier de leurs renforts avant d'être contraints, vieillards amers ou affectant d'être satisfaits, de leur céder la place. Le souvenir de leur révolte d'antan se réfugiera dans la littérature et s'estompera alors que les chercheurs continueront de s'interroger sur les effets démographiques, sociaux et politiques de leur incursion insolite dans le champ habituellement défendu de la violence, de la politique, et à plus forte raison des deux réunies.

\section{NOTES}

1. Annick Percheron, dont la (les) thèse(s) fait (font) autorité en la matière, reconnaît que l'âge représente une variable-clef mais elle souligne plus loin le caractère flou et artificiel du concept d'âge. Elle assigne à la socialisation politique d'avant l'âge adulte quatre périodes : la première se situant avant les 7-8 ans ; au cours de la dernière, après 16 ans, il ne s'agit plus que de "réorganisation, de transformations, de compléments, voire de resocialisation", bien que certaines recherches sur les phénomènes de socialisation aient porté sur de "vieux" adolescents de 16-24 ans. Annick Percheron, La socialisation politique dans l'enfance et l'adolescence, Thèse de doctorat d'Etat, I.E.P. de Paris, 1984, pp. 107-110.

2. Les Protocoles additionnels aux Conventions de Genève (1977) auxquels renvoie la Convention relative aux droits de l'enfant des Nations Unies (20/11/1989) déclarent illégal « leur recrutement (des enfants de moins de quinze ans) dans leurs forces armées (des Parties aux conflits) mais non leur participation spontanée ». En matière de conflit armé non international ils ne doivent être "ni recrutés dans les forces ou 
groupes armés, ni autorisés à prendre part aux hostilités". Néanmoins combattants éventuellement, ils bénéficient en tant que prisonniers de guerre ou internés civils d'un régime "privilégié". Ils peuvent néanmoins faire l'objet de poursuites pénales, notamment pour crimes de guerre. On notera la tendance de certains gouvernements d'étendre jusqu'à 18 ans le "statut" d'enfants-combattants. Cf. Maria Teresa Dutli, "Enfants-combattants prisonniers", Revue Internationale de la Croix Rouge, $\mathrm{n}^{\circ} 785$, sept.-oct. 1990, pp. 456-470.

3. Autrement dit : retomber en enfance.

4. Alain Touraine, Qu'est-ce que la démocratie ?, Paris, Fayard, 1994, p. 93.

5. Cf. Bertrand Badie, Guy Hermet, Politique Comparée, Paris, PUF (Coll. Thémis), 1990, p. 174. Ici même Frédéric Chauvaud écrit qu'au XXème siècle "l'enfant (...) dès sa naissance représente une menace virtuelle" ; voir infra.

6. Entretien avec Charles Melman, psychiatre, pour qui cette limite est "celle de la préservation de la vie et de sa reproduction", sans compter que "par la faute d'une sexualité biologiquement déficiente, la violence pourra être la modalité privilégiée de l'expression des tendances (1995).

7. Entretien avec Marcel Czermak, qui est directeur de la revue Journal Français de Psychiatrie, voir infra page 187.

8. Les journalistes, par contre, trouvent dans la violence juvénile un thème très vendeur. Ainsi Jean-Pierre Langellier reconnaît leur férocité sans égale", mais ajoute comme si cela ne pouvait s'expliquer autrement qu'ils sont "poussés au crime par les adultes", cf. "Enfants de Guerre", Le Monde 21/12/1993.

9. Le Monde, du 20/04/1995

10. Dans l'article précité J.P.Langellier cite à la suite les jeunes Khmers rouges, les gamins ougandais, les enfants-loups du Mozambique, les disciples galvanisés du Sentier lumineux, les jeunes Tigres tamouls, les enfants iraniens envoyés à la boucherie, les sicarios colombiens. Mentionnant le Sierra Leone, Frédéric Fritscher, cite le cas d'un jeune "démobilisé" de neuf ans. L'auteur énumère aussi d'autres pays où se pratiquent l'enrôlement juvénile : Afghanistan, Philippines, Nicaragua, Salvador, Soudan. Il aurait pu en nommer d'autres Le Monde, 23/12/1993.

11. Bernard Thomas, La croisade des Enfants, Paris, Fayard, 1973.

12. Cf. Desmond Bell, "Acts of Union", The British Journal of Sociology, XXXVIII, $n^{\circ} 2$, june, 1987, pp. 161-162.

13. Cette contestation sociale et d'abord familiale est générale, selon Piaget, chez

l'enfant de 12 ans qui "apprend à n'avoir que mépris et désintérêt pour la société réelle qu'il condamne (..) L'enfant (...) avec ses compagnons d'âge commente le présent et construit les rêves d'une société nouvelle". Ibid. p. 111.

14. Murray Last, de son coté, suggère l'idée d'une limitation d'âge "par le haut" d'une violence politique juvénile au demeurant tardive en deçà de laquelle une violence politique bien tempérée est admissible. Au delà, il vaut mieux accéder à la respectabilité islamique des "vrais" adultes. Colloque MSH 1994, Centre d'Etudes Africaines.

15. "La politique (...) est affaire d'adultes, qui cherchent à en tenir éloignés les enfants". Annick Percheron, op.cit. p. 49. 


\section{RÉSUMÉS}

Violence politique et enfance sont apparemment antinomiques. Bien que l'enfant, en effet, naturellement violent comme tout le monde, accède très précocement à une capacité politique, la pratique de celle-ci, liée au statut d'adulte, lui est interdite. Illégitime au yeux de la communauté internationale qui s'est même essayée à légiférer en la matière, la violence juvénile reste cependant pour des pouvoirs étatiques ou infra-étatiques peu regardants une ressource aisément mobilisable au service de fins politiques à la définition desquelles les enfants embrigadés demeurent étrangers. Exceptionnellement, dans des conditions de déstabilisation sociale et d'anarchie politique avancées, la violence politique des enfants revêt un caractère autonome, mais cette authentique subversion de l'ordre des choses, exposée à la reprise en main des adultes et au vieillissement même des enfants, n'a qu'une espérance de vie limitée. Quoique dépourvue d'avenir, la violence politique juvénile laisse des traces dans la société globale dont elle nourrit l'imagination et favorise les mutations.

\section{INDEX}

Mots-clés : violence, enfants, sociologie des conflits (polémologie) 